\title{
Stage-specific profiling of Plasmodium falciparum proteases using an internally quenched multispecificity protease substrate
}

\author{
Priyaranjan Pattanaik, ${ }^{\mathrm{a}}$ Bimba Jain, ${ }^{\mathrm{a}}$ Gudihal Ravindra, ${ }^{\mathrm{b}}$ Hosahudya N. Gopi ${ }^{\mathrm{b}}$ \\ Prajna P. Pal, ${ }^{\mathrm{b}}$ Hemalatha Balaram, ${ }^{\mathrm{a}}$ and Padmanabhan Balaram ${ }^{\mathrm{b}, *}$ \\ a Molecular Biology and Genetics Unit, Jawaharlal Nehru Centre for Advanced Scientific Research, Bangalore 560064, India \\ ${ }^{\mathrm{b}}$ Molecular Biophysics Unit, Indian Institute of Science, Bangalore 560012, India
}

\begin{abstract}
Novel internally quenched fluorescence peptide substrates containing sequence specific sites for cleavage by multiple proteases were designed and synthesized. The 28 and 29 residue peptides contain an N-terminal fluorescence acceptor group, 4-(4-dimethylaminophenylazo)benzoic acid (DABCYL), and a C-terminal fluorescence donor group, 5-(2-aminoethylamino)naphthalene-1sulfonic acid (EDANS). Efficient energy transfer between the donor and acceptor groups flanking the peptide sequence was achieved by incorporation of a central ${ }^{D}$ Pro-Gly segment, which serves as a conformation nucleating site, inducing hairpin formation. This multispecificity protease substrate was used to profile the proteolytic activities in the malarial parasite Plasmodium falciparum in a stage dependent manner using a combination of fluorescence and MALDI mass spectrometry. Cysteine protease activity was shown to be dominating at neutral $\mathrm{pH}$, whereas aspartic protease activity contributed predominantly to the proteolytic repertoire at acidic pH. Maximum proteolysis was observed at the trophozoite stage followed by the schizonts and the rings.
\end{abstract}

Keywords: Fluorescent protease substrate; Fluorescence resonance energy transfer; $\beta$-Hairpin peptide; Plasmodium falciparum; Malarial proteases; Mass spectrometry

Selective expression of proteases and controlled proteolytic activity is a hallmark of developmental processes in biology [1]. Plasmodium falciparum, the causative agent for the most lethal form of malaria, displays a carefully orchestrated cascade of proteolytic events throughout its life cycle, which is essential for its survival and propagation [2,3]. Proteases belonging to four major classes namely serine, aspartic, cysteine, and metalloproteases have been reported from the parasite. The entire proteolytic activity during the intraerythrocytic stage may be divided into two categories-proteolysis involved in hemoglobin degradation [4] and proteolysis involved in erythrocyte rupture and merozoite invasion of fresh erythrocytes [5]. Proteolytic activity in the parasite depends on the developmental stage and may also be localized in specialized organelles as in

\footnotetext{
${ }^{*}$ Corresponding author. Fax: +91-80-360-0683.

E-mail address: pb@mbu.iisc.ernet.in (P. Balaram).
}

the case of hemoglobin degradation, which occurs in the food vacuole at $\mathrm{pH} 5[6,7]$. Consequently, the vacuolar proteases display optimal hemoglobinase activity around this $\mathrm{pH}$. Several hemoglobinases have been identified and characterized, including four aspartic proteases-plasmepsin I, II, IV, and histo-aspartic protease (HAP) [8-11], three cysteine proteases-falcipain 1, 2 , and 3 [12-14], and at least one metalloprotease-falcilysin [15]. The second pool of proteolysis, which is related with erythrocyte rupture and invasion, involves the cytosolic environment, where the $\mathrm{pH}$ is near neutral. Thus, cellular proteolytic activity can also be modulated by local environmental conditions.

We describe in this report a new approach to the deconvolution of the repertoire of proteolytic activity in the intraerthrocytic stage of $P$. falciparum in a class specific manner at different stages of parasite growth. The present study is based on the use of designed, internally quenched fluorescent 'universal' protease 
substrates, which contain several specific cleavage sites. Application of a combination of fluorescence and mass spectral analysis permits the profiling of $P$. falciparum proteolysis.

\section{Materials and methods}

Synthesis of the peptide substrate. Internally quenched peptides were synthesized following the method described by Luker et al. [8] with modifications. The peptide 1 (Fig. 1) was assembled by Fmoc solid phase chemistry on a flow synthesizer. Peptide $\mathbf{2}$ was obtained as a deletion product, which was purified and characterized. All amino acids were protected at the N-terminus with the Fmoc group (NovaBiochem). The side chains of Asp, Glu, and Ser were protected with $t$-Bu group, Arg with Mtr group, Cys with trityl group, and Lys with Boc group. The coupling reaction proceeded using the Opfp esters of the protected amino acids on PAC-PEG-PS resin (Perspective Biosystems). Fmoc deprotections were done with $20 \%$ piperidine in dimethylformamide. The resin assembled peptide was subsequently cleaved in $94 \%$ TFA, containing 5\% anisole and $1 \%$ ethanedithiol as cation scavengers. The isolated crude peptide was dissolved in a 9:1 mixture of THF and water. 5-(2-(iodoacetyl)aminoethyl)amino naphthalene-1-sulfonate (IAEDANS) was added five times in excess to the solution at $\mathrm{pH}$ 8.0. The purification of the peptide was achieved by reverse phase HPLC on a $\mathrm{C}_{18}$ column with a linear gradient of acetonitrile containing $0.1 \%$ TFA. The purified peptides were characterized by MALDI-TOF mass spectrometry-peptide 1: $M_{\text {calc }}=3665, M_{\text {obs }}=3689[\mathrm{M}+\mathrm{Na}]^{+}$; peptide 2: $M_{\text {calc }}=3509, M_{\text {obs }}=3532[\mathrm{M}+\mathrm{Na}]^{+}$.

Parasite culture, isolation, and assay for protease activity. P. falciparum (strain T9/106) culture grown by the candle jar method [16] was synchronized by treatment with sorbitol. Cells isolated by saponin treatment at the ring, trophozoite, and schizont stages were suspended in the buffer to be used for protease assay- $100 \mathrm{mM}$ sodium phosphate ( $\mathrm{pH} 7.2$ ) or $100 \mathrm{mM}$ sodium acetate ( $\mathrm{pH} 4.8$ ), containing $5 \mathrm{mM}$ DTT and sonicated briefly over ice. The lysed suspension was clarified of hemozoin pigments by centrifuging at $20,000 \mathrm{~g}$ for $15 \mathrm{~min}$. The supernatant was directly used for the protease assay avoiding storage.
Specific inhibitors used in the assay were $10 \mu \mathrm{M}$ E64 ( $N$-(trans-epoxysuccinyl)-L-leucine-4-guanidinobutylamide), $10 \mu \mathrm{M}$ PMSF (phenylmethylsulfonyl fluoride), $10 \mu \mathrm{M}$ pepstatin A, and $1 \mathrm{mM}$ EDTA (ethylenediaminotetraacetic acid). Prior to the assay, the cell lysates were incubated for $30 \mathrm{~min}$ in the presence of the inhibitors. The assay was done in $500 \mu \mathrm{l}$ of $100 \mathrm{mM}$ buffer (sodium phosphate, $\mathrm{pH} \mathrm{7.2,} \mathrm{or}$ sodium acetate, $\mathrm{pH} 4.8$ ), with $5 \mathrm{mM}$ DTT and $1.8 \mu \mathrm{M}$ peptide substrate. Proteolysis was monitored as increase in emission intensity with time at $490 \mathrm{~nm}$, following excitation at $335 \mathrm{~nm}$. A Perkin-Elmer LS55B fluorimeter was used with excitation and emission bandpass kept at 7 and $10 \mathrm{~nm}$, respectively.

MALDI mass spectrometry. For recording MALDI spectra, the reaction volume was brought down to $50 \mu \mathrm{l}$ and the reaction was stopped by freezing in liquid nitrogen. MALDI spectra were recorded in positive ion mode using a KOMPACT SEQ spectrometer (KratosShimadzu Analytical, Manchester, UK). The matrices used for positive ion mode detection were $\alpha$-cyano-4-hydroxycinnamic acid and 3,5-dimethoxy-4-hydroxycinnamic acid in $60 \%$ acetonitrile containing $0.1 \%$ TFA. Routinely, $0.5 \mu \mathrm{l}$ of matrix was mixed with $0.5 \mu \mathrm{l}$ of the reaction solution on a MALDI plate for mass spectral analysis. Each sample was spotted thrice and spectra were recorded for each spot. After proteolysis, the $\mathrm{N}$-terminal fragments were readily detectable. C-terminal fragments ionized poorly under the conditions used, presumably due to the dansyl sulfonic acid moiety, which forms tight ion pairs.

\section{Results and discussion}

Fig. 1 shows the sequences of two substrates with specific cleavage points indicated. Peptide 2 lacks a second trypsin site, which is present in peptide $\mathbf{1}$ (-MetArg-Glu-). The peptides incorporate a central DPro-Gly segment, which facilitates hairpin formation $[17,18]$, thus permitting population of a range of conformations in which the fluorescent donor and acceptor groups lie at distances that facilitate quenching by fluorescence resonance energy transfer (FRET) [19-21]. This strategy

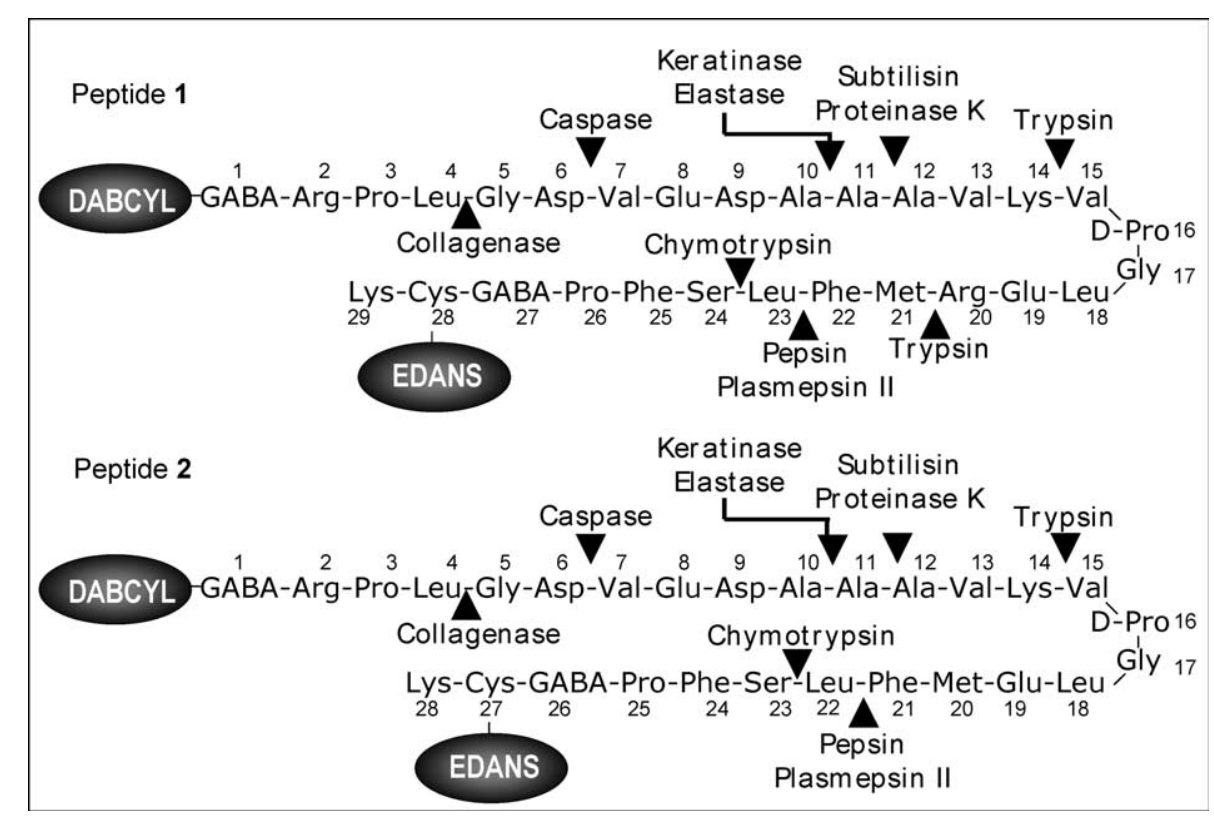

Fig. 1. Sequences of the universal protease substrates $\mathbf{1}$ and 2. Potential protease cleavage sites are indicated. 
permits the linear assembly of a number of specific protease cleavage sites into a single substrate molecule. Thus far, the use of internally quenched fluorescent substrates has been limited to relatively short peptide sequences (812 residues), since the efficiency of quenching diminishes rapidly as the length of the intervening polypeptide increases [19]. FRET based protease assays have been used in the literature for monitoring many therapeutically and physiologically important enzymes like the retroviral proteases [22], renin [23], hepatitis A3C protease [24], interleukin $1 \beta$ converting enzyme (ICE) [25], Leishmania surface metalloprotease [26], anthrax lethal factor [27], Candida albicans aspartic protease [28], papain [29], and the plasmodial protease-plasmepsin II $[8,30,31]$. The chromophores chosen as fluorescence donor and acceptor are 5-(2-aminoethylamino)naphthalene-1-sulfonic acid (EDANS) and 4-(4-dimethylaminophenylazo)benzoic acid (DABCYL), respectively. The fluorescence emission band of EDANS $(490 \mathrm{~nm})$ displays excellent overlap with the broad visible absorption band of DABCYL. The Fórster distance $\left(R_{0}\right)$ for these two chromophores is estimated to be about $33 \AA$ [22].

Plasmodium falciparum cells were isolated from highly synchronized in vitro culture at three morphologically distinct stages namely_ring, trophozoite, and schizont. Protease activities in the lysates of these cells were monitored at $\mathrm{pH} 7.2$ and 4.8 using peptide $\mathbf{2}$ (Fig. 1). Measurement of proteolysis by members of any one particular class was achieved by including a combination of class-specific inhibitors in the reaction mix-

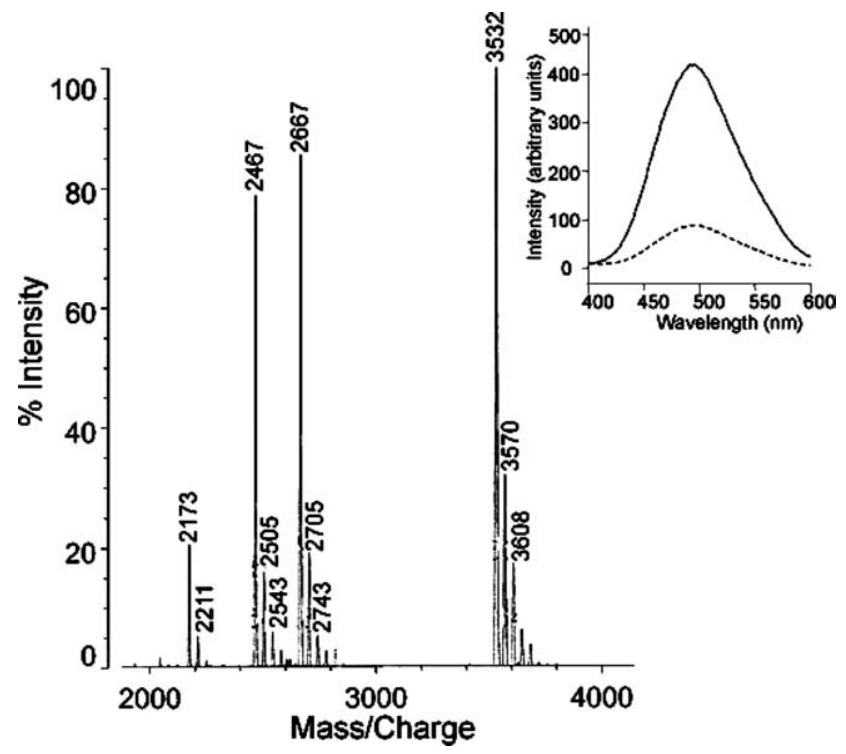

Fig. 2. MALDI mass spectrum of peptide 2 treated with $P$. falciparum trophozoite extract at $\mathrm{pH} 7.2$ in the absence of any inhibitors (see Materials and methods for details). All the peaks are marked by the corresponding masses (Da). Mass of 3532 corresponds to the intact peptide. Inset: Emission spectra of peptide $\mathbf{2}$ at $\mathrm{pH} 7.2$ (broken line) and after addition of $P$. falciparum trophozoite extract (solid line). Excitation: $335 \mathrm{~nm}$. ture. Inhibitors used were E64 cysteine protease; $\mathrm{PMSF}$ - serine protease, pepstatin A-aspartic protease, and EDTA-metalloprotease. Fig. 2 shows representative fluorescence and mass spectra obtained upon cleavage of peptide 2 by a trophozoite extract. Fig. 3 summarizes the results of assays of protease activities from different developmental stages of the parasite, expressed as an increase in fluorescence intensity of EDANS. In each case, inclusion of all four inhibitors resulted in no increase in fluorescence, while maximum activity was obtained in the absence of any inhibitor. This validated the use of such an assay system and confirmed the ability of these four inhibitors, working in
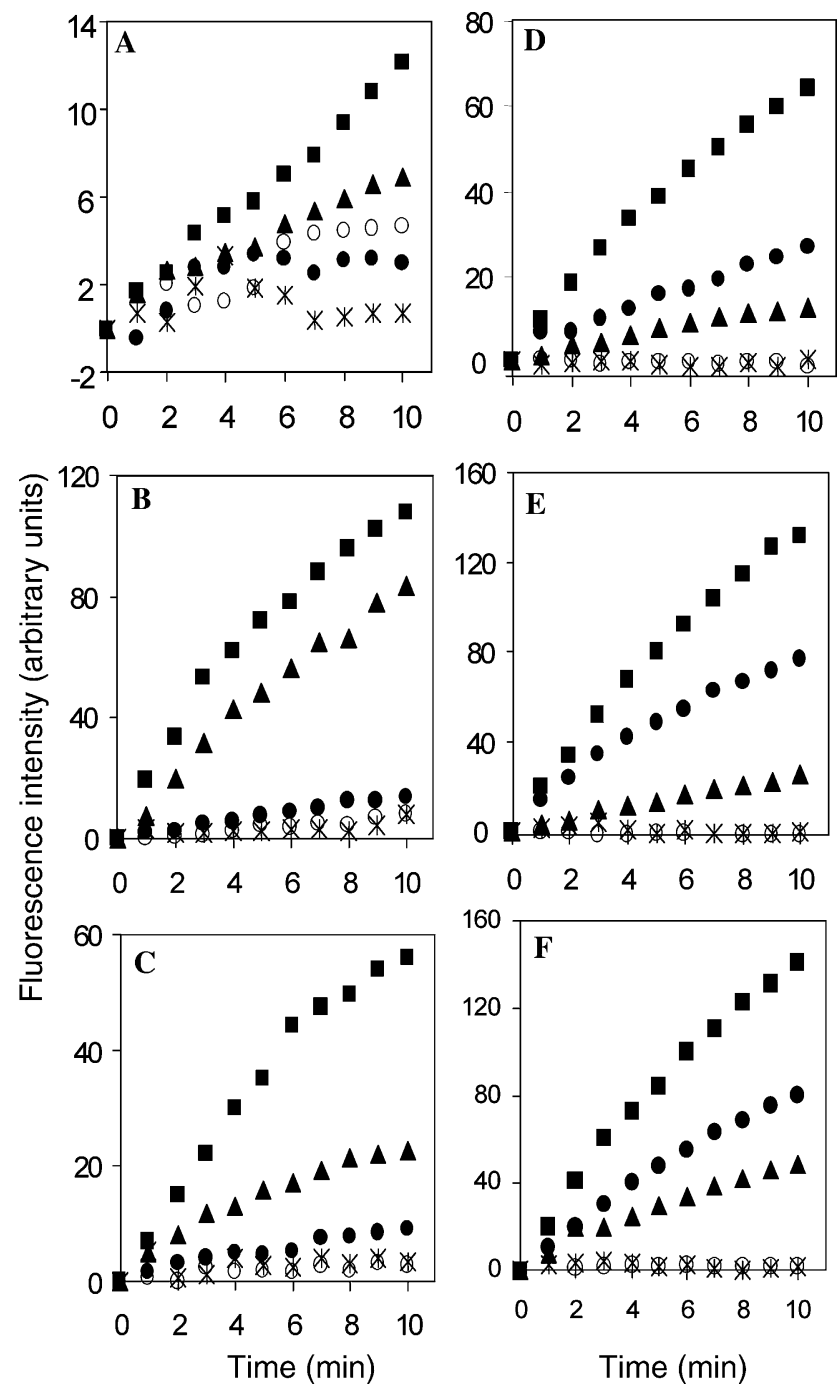

Fig. 3. Protease activity in cell lysates of $P$. falciparum at $\mathrm{pH} 7.2$ (left panel) and 4.8 (right panel). Cell lysates at ring (A and D), trophozoite (B and $\mathrm{E}$ ), and schizont ( $\mathrm{C}$ and $\mathrm{F}$ ) stages were assayed using peptide 2 in the presence of E64, pepstatin A, PMSF, and EDTA (asterisk); E64, PMSF, and EDTA (closed circle); pepstatin A, PMSF, and EDTA (triangle); and E64, pepstatin A, and EDTA (open circle) or in the absence of any inhibitor (square). Proteolysis is presented here as an increase in fluorescence emission intensity with time at $490 \mathrm{~nm}$ following excitation at $335 \mathrm{~nm}$. 
tandem, to inhibit the entire proteolytic machinery. Differences in total protease activities between acidic and neutral $\mathrm{pH}$ were minimum in the trophozoite stage (Figs. 3B and E). There was significantly higher protease activity at acidic $\mathrm{pH}$ compared to the neutral $\mathrm{pH}$ in the schizont stage (Figs. 3C and F). The ring stage however presented a stark difference in proteolytic activity at the two $\mathrm{pH}$ values, having almost negligible total protease activity at pH 7.2 (Figs. 3A and D). This observation could be substantiated by taking into account the relative ratio of cytoplasm to food vacuole. In the ring stage the parasite cell is dominated by the presence of the food vacuole, which leaves only a thin smear of cytoplasm around it. This stage also marks the cellular preparation for large-scale hemoglobin degradation in the food vacuole and hence, the upregulation of acidic protease activity (Fig. 3D). Cytoplasmic proteases are largely involved with rupture and invasion and both these processes are inconspicuous in the ring stage. This may be one of the reasons for the very low levels of neutral protease activity in the ring stage (Fig. 3A). Also, minimum overall protease activity in the ring stage could be a reflection of low cellular protein content. The trophozoite stage is marked by extensive hemoglobin degradation, combined with cellular growth. The appearance of significant activity corresponding to neutral proteases may be attributed to increased cytoplasmic content at this stage (Fig. 3B). This also indicates the beginning of cellular processes that lead to rupture of the RBC at the end of the intraerythrocytic cycle. The schizont stage with distinct daughter cells is decorated by the largest parasitoporous vacuole that occupies most of the intracellular chamber inside RBC. Complete disappearance of the food vacuole is seen in this stage. Hence, comparable acidic protease activity between the schizont and trophozoite stages was unex- pected. In all the three stages aspartic protease activity contributed maximally towards total activity, with significant contribution from the cysteine proteases at $\mathrm{pH}$ 4.8, whereas the scenario was reversed at neutral $\mathrm{pH}$. Recent reports emphasizing cysteine protease activity involved in the process of rupture [32] and invasion [33] validate the presence of high level of cysteine protease activity at $\mathrm{pH} 7.2$ in trophozoite and schizont stages (Figs. 3B and C).

Two serine proteases resembling subtilisin-PfSub1[34] and PfSub-2 [35] have been cloned and characterized from $P$. falciparum in addition to evidences for the presence of other serine protease activities. Our assay did not detect serine and metalloprotease activities in any significant amount. Two factors may account for this observation: (i) though important from a cellular point of view, these proteases may not be present in abundance or (ii) the substrate used does not contain a suitable site for cleavage by these proteases, although model studies demonstrate ready cleavage by common serine proteases like trypsin, chymotrypsin, and subtilisin, and also by metalloenzymes like carboxypeptidase (data not shown). It should also be emphasized that earlier studies suggested that serine protease activity is detectable only after treatment with phospholipase, which releases the protease from the membrane anchor [36]. One possible reason for the absence of serine protease activity in our assay could be that the protease remained attached to the membrane fragments and hence, rendered inactive under our experimental conditions.

Cleavage sites in the peptide under diverse conditions were established by MALDI mass spectrometry of the cleavage products. Mass spectra determined after incubation with parasite lysate showed the presence of satellite peaks, which differed in mass of $38 \mathrm{Da}$ from the

Table 1

Sites cleaved by $P$. falciparum cell extract at the trophozoite ${ }^{a}$ stage identified by MALDI mass spectrometry

\begin{tabular}{|c|c|c|c|c|}
\hline Protease class & $\mathrm{pH}$ & Site of cleavage & [Fragment], Expected mass $\left(\mathrm{M}^{+}\right)$ & Observed mass ${ }^{\mathrm{e}}\left(\mathrm{MH}^{+}\right)$ \\
\hline \multirow[t]{2}{*}{ Aspartic ${ }^{b}$} & 7.2 & F21-L22 & [1-21], 2449 & 2468 \\
\hline & 4.8 & F21-L22 & [1-21], 2449 & 2468 \\
\hline \multirow[t]{4}{*}{ Cysteine $^{c}$} & 7.2 & $\mathrm{~S} 23-\mathrm{F} 24$ & [1-23], 2649 & 2664 \\
\hline & & L18-E19 & [1-18], 2171 & 2170 \\
\hline & 4.8 & S23-F24 & [1-23], 2649 & 2662 \\
\hline & & L18-E19 & {$[1-18], 2171$} & 2169 \\
\hline \multirow{6}{*}{ All proteases ${ }^{\mathrm{d}}$} & 7.2 & F21-L22 & [1-21], 2450 & 2467 \\
\hline & & S23-F24 & {$[1-23], 2649$} & 2667 \\
\hline & & L18-E19 & {$[1-18], 2171$} & 2173 \\
\hline & 4.8 & F21-L22 & {$[1-21], 2450$} & 2467 \\
\hline & & S23-F24 & [1-23], 2649 & 2667 \\
\hline & & L18-E19 & {$[1-18], 2171$} & 2173 \\
\hline
\end{tabular}


parent and may be assigned to multiple $\mathrm{K}^{+}$adducts. It may be noted that during preparation of the parasite lysates PBS was used, which has a potassium ion concentration of about $45 \mathrm{mM}$. The parent peaks are assigned to the protonated species $\left[\mathrm{MH}^{+}\right]$. Furthermore, the fragments appear to be oxidized to the sulfoxide, resulting in an adduct mass of $16 \mathrm{Da}$. The peaks obtained under a particular condition of assay were essentially identical in all three stages of parasite growth. Presence of all four inhibitors resulted in complete absence of any peak corresponding to substrate fragments. Table 1 shows the sites of cleavage determined from MALDI mass spectral analysis at the trophozoite stage. Under all conditions the aspartic proteases were able to cleave the substrate only at positions F21-L22. This site corresponds to the reported plasmepsin II [37] cleavage preference. Plasmepsin I and HAP were also reported to possess the same cleavage preference. Cysteine proteases cleaved the substrate at two sites L18-E19 and S23-F24 under all conditions. All the three cleavage sites were detected when the assay was done in the absence of any inhibitor.

Sequencing of the malarial genome reveals the presence of about 29 genes annotated as proteases [38]. Ten genes coding for aspartic proteases have been identified [39] of which seven were shown to be expressed in the intraerythrocytic stages [11]. Similarly, there are several genes in the $P$. falciparum genome putatively coding for cysteine proteases other than the well-characterized falcipains. This raises questions on the existence of more than one enzyme with seemingly similar substrate specificity that performs the same cellular function such as hemoglobin degradation. Most proteases display sharp dependence on $\mathrm{pH}$ for proteolysis. In our assay, both cysteine and aspartic protease activities are observed at both acidic and neutral $\mathrm{pH}$. Though surprising, this observation is consistent with the reports showing neutral $\mathrm{pH}$ activity for both plasmepsin II [40] and falcipain 2 [41]. Our data also suggest that there is no alteration in substrate recognition by both classes of proteases between acidic and neutral $\mathrm{pH}$. Whether each member of a class of proteases is programmed to be localized in either the food vacuole or in the cytoplasm, or the same protease functions in both compartments needs to be answered.

In summary, we have presented here a novel FRET based protease substrate designed to harbor cleavage sites preferred by multiple proteases. Limitations in the length of the peptide from the point of view of efficient internal quenching have been overcome by inducing the peptide to fold into a $\beta$-hairpin using a centrally positioned conformation nucleating site. This substrate has been used to profile the protease activities in a complex mixture like a $P$. falciparum cell extract. Proteases are central to the survival of many disease-causing parasites, which make them attractive targets for therapeutic in- tervention [1]. Identification of these proteases and their characterization is often hampered by the lack of information regarding their catalytic preferences and limited availability from the natural source. Our approach of using a universal substrate not only presents a highly sensitive way of monitoring multiple proteases of varied specificities, but also provides information regarding the nature of the cleavage sequence preferred by individual enzymes. It is clear that this approach also lends itself to large-scale screening of proteases using a combination of automated fluorescence and mass spectrometric methods.

\section{Acknowledgments}

This research was supported by grants from Council for Scientific and Industrial Research (CSIR), Government of India, and a programme support grant in the area of Molecular Diversity and Design, Department of Biotechnology (DBT), Government of India.

\section{References}

[1] M. Klemba, D.E. Goldberg, Biological roles of proteases in parasitic protozoa, Annu. Rev. Biochem. 71 (2002) 275-305.

[2] P. Pattanaik, J. Raman, H. Balaram, Perspectives in drug design against malaria, Curr. Top. Med. Chem. 2 (2002) 483-505.

[3] P.L. Olliaro, Y. Yuthavong, An overview of chemotherapeutic targets for antimalarial drug discovery, Pharmacol. Ther. 81 (1999) 91-110.

[4] S.E. Francis, D.J. Sullivan Jr., D.E. Goldberg, Hemoglobin metabolism in the malaria parasite Plasmodium falciparum, Annu. Rev. Microbiol. 51 (1997) 97-123.

[5] M.J. Blackman, Proteases involved in erythrocyte invasion by the malaria parasite: function and potential as chemotherapeutic target, Curr. Drug Targets 1 (2000) 59-83.

[6] I.Y. Gluzman, S.E. Francis, A. Oksman, C.E. Smith, K.L. Duffin, D.E. Goldberg, Order and specificity of the Plasmodium falciparum hemoglobin degradation pathway, J. Clin. Invest. 93 (1994) 1602-1608.

[7] D.E. Goldberg, Plasmodial hemoglobin degradation: an ordered pathway in a specialized organelle, Infect. Agents Dis. 1 (1992) 207-211.

[8] K.E. Luker, S.E. Francis, I.Y. Gluzman, D.E. Goldberg, Kinetic analysis of plasmepsins I and II, aspartic proteases of the Plasmodium falciparum digestive vacuole, Mol. Biochem. Parasitol. 79 (1996) 71-78.

[9] R.P. Moon, L. Tyas, U. Certa, K. Rupp, D. Bur, C. Jacquet, H. Matile, H. Loetscher, F. Grueninger-Leitch, J. Kay, B.M. Dunn, C. Berry, R.G. Radley, Expression and characterisation of plasmepsin I from Plasmodium falciparum, Eur. J. Biochem. 244 (1997) 552-560.

[10] D.M. Wayatt, C. Berry, Activity and inhibition of plasmepsin IV, a new aspartic proteinase from the malaria parasite, Plasmodium falciparum, FEBS Lett. 513 (2002) 159-162.

[11] R. Banerjee, J. Liu, W. Beatty, L. Pelosof, M. Klemba, D.E. Goldberg, Four plasmepsins are active in the Plasmodium falciparum food vacuole, including a protease with an active-site histidine, Proc. Natl. Acad. Sci. USA 99 (2002) 990-995.

[12] F. Salas, J. Fichmann, G.K. Lee, M.D. Scott, P.J. Rosenthal, Functional expression of falcipain, a Plasmodium falciparum 
cysteine proteinase, supports its role as a malarial hemoglobinase, Infect. Immun. 63 (1995) 2120-2125.

[13] B.R. Shenai, P.S. Sijwali, A. Singh, P.J. Rosenthal, Characterization of native and recombinant falcipain-2, a principal trophozoite cysteine protease and essential hemoglobinase of Plasmodium falciparum, J. Biol. Chem. 275 (2000) 29000-29010.

[14] P.S. Sijwali, B.R. Shenai, J. Gut, A.J. Singh, P.J. Rosenthal, Expression and characterization of the Plasmodium falciparum haemoglobinase falcipain-3, Biochem. J. 360 (2001) 481-489.

[15] K.K. Eggleson, K.L. Duffin, D.E. Goldberg, Identification and characterization of falcilysin, a metallopeptidase involved in hemoglobin catabolism within the malaria parasite Plasmodium falciparum, J. Biol. Chem. 274 (1999) 32411-32417.

[16] W. Trager, J.B. Jensen, Human malaria parasites in continuous culture, Science 193 (1976) 673-675.

[17] I.L. Karle, S.K. Awasthi, P. Balaram, A designed beta-hairpin peptide in crystals, Proc. Natl. Acad. Sci. USA 93 (1996) 8189 8193.

[18] J. Venkatraman, S.C. Shankaramma, P. Balaram, Chem. Rev. 101 (2001) 3131-3152.

[19] L. Stryer, Fluorescence energy transfer as a spectroscopic ruler, Annu. Rev. Biochem. 47 (1978) 819-846.

[20] R.H. Fairclough, C.R. Cantor, The use of singlet-singlet energy transfer to study macromolecular assemblies, Methods Enzymol. 48 (1978) 347-379.

[21] H.C. Cheung, in: J.R. Lakowicz (Ed.), Topics in Fluorescence Spectroscopy, vol. 2, Plenum, New York, 1991, pp. 218-286.

[22] E.D. Matayoshi, G.T. Wang, G.A. Krafft, J. Erickson, Novel fluorogenic substrates for assaying retroviral proteases by resonance energy transfer, Science 247 (1990) 954-958.

[23] G.T. Wang, C.C. Chung, T.F. Holzman, G.A. Krafft, Continuous fluorescence assay of renin activity, Anal. Biochem. 210 (1993) 351-359.

[24] M. Taliani, E. Bianchi, F. Narjes, M. Fossatelli, A. Urbani, C. Steinkuhler, R.. DeFrancesco, A. Pessi, A continuous assay of hepatitis $\mathrm{C}$ virus protease based on resonance energy transfer depsipeptide substrates, Anal. Biochem. 240 (1996) 60-67.

[25] M.W. Pennington, N.A. Thornberry, Synthesis of a fluorogenic interleukin-1 beta converting enzyme substrate based on resonance energy transfer, Pept. Res. 7 (1994) 72-76.

[26] J. Bouvier, P. Schneider, B. Malcolm, A fluorescent peptide substrate for the surface metalloprotease of Leishmania, Exp. Parasitol. 76 (1993) 146-155.

[27] R.T. Cummings, S.P. Salowe, B.R. Cunningham, J. Wiltsie, Y.W. Park, L.M. Sonatore, D. Wisniewski, C.M. Douglas, J.D. Hermes, E.M. Scolnick, A peptide-based fluorescence resonance energy transfer assay for Bacillus anthracis lethal factor protease, Proc. Natl. Acad. Sci. USA 99 (2002) 6603-6606.

[28] J.O. Capobianco, C.G. Lerner, R.C. Goldman, Application of a fluorogenic substrate in the assay of proteolytic activity and in the discovery of a potent inhibitor of Candida albicans aspartic proteinase, Anal. Biochem. 204 (1992) 96-102.
[29] C. Garcia-Echeverria, D.H. Rich, New intramolecularly quenched fluorogenic peptide substrates for the study of the kinetic specificity of papain, FEBS Lett. 297 (1992) 100-102.

[30] A.M. Silva, A.Y. Lee, S.V. Gulnik, P. Maier, J. Collins, T.N. Bhat, P.J. Collins, R.E. Cachau, K.E. Luker, I.Y. Gluzman, S.E. Francis, A. Oksman, D.E. Goldberg, J.W. Erickson, Structure and inhibition of plasmepsin II, a hemoglobin-degrading enzyme from Plasmodium falciparum, Proc. Natl. Acad. Sci. USA 93 (1996) 10034-10039.

[31] H.N. Gopi, G. Ravindra, P.P. Pal, P. Pattanaik, H. Balaram, P. Balaram, Proteolytic stability of beta-peptide bonds probed using quenched fluorescent substrates incorporating a hemoglobin cleavage site, FEBS Lett. 535 (2003) 175-178.

[32] B.L. Salmon, A. Oksman, D.E. Goldberg, Malaria parasite exit from the host erythrocyte: a two-step process requiring extraerythrocytic proteolysis, Proc. Natl. Acad. Sci. USA 98 (2001) 271-276.

[33] D.C. Greenbaum, A. Baruch, M. Grainger, Z. Bozdech, K.F. Medzihradszky, J. Engel, J. DeRisi, A.A. Holder, M. Bogyo, A role for the protease falcipain 1 in host cell invasion by the human malaria parasite, Science 298 (2002) 2002-2006.

[34] M. Sajid, C. Withers-Martinez, M.J. Blackman, Maturation and specificity of Plasmodium falciparum subtilisin-like protease-1, a malaria merozoite subtilisin like serine protease, J. Biol. Chem. 275 (2000) 631-641.

[35] F. Hackett, M. Sajid, C. Withers-Martinez, M. Grainger, M.J. Blackman, PfSUB-2: a second subtilisin-like protein in Plasmodium falciparum meroziotes, Mol. Biochem. Parasitol. 103 (1999) 183-195.

[36] C. Braun-Breton, T.L. Rosenberry, L.P. da Silva, Induction of the proteolytic activity of a membrane protein in Plasmodium falciparum by phosphatidyl inositol-specific phospholipase C, Nature 332 (1988) 457-459.

[37] D.E. Goldberg, A.F. Slater, R. Beavis, B. Chait, A. Cerami, G.B. Henderson, Hemoglobin degradation in the human malaria pathogen Plasmodium falciparum: a catabolic pathway initiated by a specific aspartic protease, J. Exp. Med. 173 (1991) 961-969.

[38] PlasmoDB: An integrative database of the Plasmodium falciparum genome, Nucleic Acids Res. 29 (2001) 66-69.

[39] G.H. Coombs, D.E. Goldberg, M. Klemba, C. Berry, J. Kay, J.C. Mottram, Aspartic proteases of Plasmodium falciparum and other parasitic protozoa as drug targets, Trends Parasitol. 17 (2001) $532-537$.

[40] S. Le Bonniec, C. Deregnaucourt, V. Redeker, R. Banerjee, P. Grellier, D.E. Goldberg, J. Schrevel, Plasmepsin II, an acidic hemoglobinase from the Plasmodium falciparum food vacuole, is active at neutral $\mathrm{pH}$ on the host erythrocyte membrane skeleton, J. Biol. Chem. 14 (1999) 14218-14223.

[41] M. Dua, P. Raphael, P.S. Sijwali, P.J. Rosenthal, M. Hanspal, Recombinant falcipain-2 cleaves erythrocyte membrane ankyrin and protein, Mol. Biochem. Parasitol. 116 (2001) 95-99. 